\title{
Examination of Latent Test Anxiety Profiles in a Sample of U.S. Adolescents
}

\author{
Patricia A. Lowe ${ }^{1}$ \\ ${ }^{1}$ Department of Educational Psychology, University of Kansas, Lawrence, Kansas, USA \\ Correspondence: Patricia A. Lowe, Department of Educational Psychology, University of Kansas, Lawrence, KS, \\ 66045, USA. Tel: 1-785-864-9710. E-mail: tlowe@ku.edu
}

Received: July 16, 2020

Accepted: September 9, 2020

Online Published: January 25, 2021

doi:10.5539/ies.v14n2p12

URL: https://doi.org/10.5539/ies.v14n2p12

\begin{abstract}
The present study examined latent test anxiety profiles in a sample of 592 U.S. adolescents in grades 6-12 using latent profile analysis (LPA). The adolescents were administered a multidimensional measure of test anxiety in their schools. The results of LPA indicated that a three-profile test anxiety model provided the best fitting model. The three latent test anxiety subgroups were named low, medium, and high test anxiety. In addition, grade-level and gender were added as covariates to the model and LPA was performed again. Grade-level and gender were found to differentially predict membership in the latent test anxiety subgroups, with females more likely to be in the high latent test anxiety subgroup than in the medium and low latent test anxiety subgroups and middle school students were more likely to be in the high latent test anxiety subgroup than in the low latent test anxiety subgroup. Middle school students were also more likely to be in the medium latent test anxiety subgroup than in the low latent test anxiety subgroup. Implications for the development of measures, treatment, and prevention of test anxiety in the U.S. adolescent population are discussed.
\end{abstract}

Keywords: adolescents, gender differences, grade-level differences, latent profile analysis, test anxiety

\section{Introduction}

Testing is a common occurrence in schools in the United States and abroad (Bodas \& Ollendick, 2005; Bodas, Ollendick, \& Sovani, 2008; Lowe et al., 2008). Students' test scores are used to evaluate the quality of schools, to assist in educational decision making, and to determine whether students are meeting educational expectations (Every Student Succeeds Act, 2015; Jacob, Decker, \& Timmerman Lugg, 2016). Many students in these evaluative situations experience test anxiety. Test anxiety is the cognitive, behavioral, and physiological symptoms students experience in evaluative situations (Bodas et al., 2008; Lowe et al., 2008; Zeidner, 2014). Test anxiety has been reported to be associated with poorer performance on standardized tests and lower classroom grades (Chapell et al., 2005; von der Embse, Jester, Roy, \& Post, 2018), working memory difficulties (Mowbray, 2012), lower self-efficacy (von der Embse et al., 2018), and higher rates of absenteeism and dropout (Cizek \& Burg, 2006; Wild, Hofer, \& Pekrun, 2006; Yousefi, 2012).

The construct of test anxiety consists of many different dimensions (Lowe et al., 2008; von der Embse, Mata, Segool, \& Scott, 2014; Zeidner, 1998). According to Lowe et al.'s (2008) biopsychosocial model of test anxiety, test anxiety develops through the interplay of a student's intrapersonal characteristics and one's environmental contexts along with an individual's appraisal of a test as threatening. The magnitude of the threat then affects the level and type of anxiety experienced as well as its symptom presentation. Symptoms of test anxiety include cognitive, behavioral, and physical symptoms. The Test Anxiety Measure for Adolescents (TAMA) designed specifically for secondary students in grades 6-12 in the United States and consists of cognitive (cognitive interference, social concerns, and worry), behavioral (task irrelevant behaviors), and physical (physiological hyperarousal) symptoms was developed based on Lowe et al.'s (2008) biopsychosocial model of test anxiety and the data from this measure will be used to perform latent profile analysis (LPA) in the present study. Few studies have been conducted in the field of test anxiety using LPA.

\subsection{Latent Profile Analysis}

LPA is a sophisticated statistical procedure that has become of more interest to researchers in recent years (Woo, Jebb, Tay, \& Parrigon, 2018). LPA is a type of mixture model approach used to identify smaller latent subgroups or 
classes from a larger more diverse population based on a set of variables (Carey, Devine, Hill, \& Szücs, 2017; Spurk, Hirschi, Wang, Valero, \& Kauffeld, 2020; Vermunt, \& Magidson, 2002). The individuals within a latent subgroup are purported to share a certain pattern, such as a certain test anxiety pattern, and this latent subgroup is then compared to other latent subgroups based on the patterns and levels of the latent variables of interest (Collins \& Lanza, 2013; Mammarella, Donolato, Caviola, \& Giofré, 2018). In addition, covariates can be added to LPA models to determine whether the covariates can differentially predict membership in the latent subgroups (Collins \& Lanza, 2013; Spurk et al., 2020; Wang \& Hanges, 2011). The results of LPA can be informative in the areas of scale development, treatment, and prevention (Carey et al., 2017; Mammarella et al., 2018; von der Embse et al., 2014). In the present study, latent test anxiety profiles will be examined with a multidimensional measure of test anxiety developed specifically for U. S. adolescents. To the author's knowledge, there has been no LPA study conducted that has examined latent test anxiety profiles using a multidimensional measure of test anxiety developed in the United States for adolescents and includes the cognitive dimensions of worry, cognitive interference, and social concerns, a physical (i.e., physiological hyperarousal) dimension, and a behavioral (i.e., task irrelevant behaviors) dimension. It is imperative to include multiple dimensions of test anxiety in LPA studies in order to develop a better and more comprehensive understanding of these profiles, so we can advance our knowledge in the field of test anxiety.

\subsection{Covariates, Latent Profile Analysis, and Test Anxiety}

Grade-level and gender can serve as covariates in LPA models. Grade-level and gender differences have been reported in the test anxiety literature (Hembree, 1988; Knappe et al., 2011; Putwain \& Daly, 2014; Ringeisen \& Raufelder, 2015; von der Embse et al., 2018; Wigfield \& Eccles, 1989). Von der Embse et al. (2018) and Wigfield and Eccles (1989) examined age/grade-level differences in test anxiety and found test anxiety to peak in students during their middle school/junior high school years and then level off during their high school years. On the other hand, Knappe et al. (2011) found test anxiety fears continue to increase in students through their postsecondary school years, peaking around the age of 21 . In contrast, gender differences in test anxiety tend to be clearer with females consistently reporting higher levels of test anxiety than males (Hembree, 1988; Putwain \& Daly, 2014; Ringeisen \& Raufelder, 2015; von der Embse et al., 2018). To the author's knowledge no study has included grade-level and gender as covariates in LPA models using a measure that includes cognitive (i.e., worry, social concerns, cognitive interference), physical (i.e., physiological hyperarousal), and behavioral (i.e., task irrelevant behaviors) dimensions of test anxiety.

\subsection{Aims and Hypotheses}

Three specific aims were associated with the current study. The first aim was to examine heterogeneity in self-reported test anxiety among a sample of U.S. secondary students using different anxiety profiles. The results of a pilot study conducted with only 11th grade U.S. students on a three-dimensional measure of test anxiety developed in another country found a three-profile model of test anxiety as the best fitting model (von der Embse et al., 2014). Therefore, it was hypothesized that a three-profile model of test anxiety would be found in the current study with adolescents in grades 6-12 on a different multidimensional measure of test anxiety developed in the United States. The second aim of the study was to determine whether gender could differentially predict membership in any of the latent test anxiety subgroups identified in the adolescent sample. Based on gender differences reported in the literature (Hembree, 1988; Putwain \& Daly, 2014; Ringeisen \& Raufelder, 2015; von der Embse et al., 2018), it was hypothesized that females would be more likely to be found in the higher latent test anxiety subgroup(s) than males. The third aim of the study was to determine whether grade-level could differentially predict membership in any of the latent test anxiety subgroups identified in a sample of secondary students. Research has shown that grade-level differences do exist (Knappe et al., 2011; von der Embse et al., 2018; Wigfield \& Eccles, 1989) however; it is unclear whether middle school students or high school students would experience higher levels of test anxiety. No hypothesis was proposed as to whether middle school students or high school students would be more likely to be found in the higher latent test anxiety subgroup(s) due to the equivocal findings reported in the literature.

\section{Method}

\subsection{Participants}

Students $(n=592)$ in 9 middle schools and high schools from the midwestern region of the United States participated in the present study. There were $248(41.9 \%)$ males and $344(58.1 \%)$ females, aged 11 to 19 years ( $M$ $=13.66, S D=2.04)$. The students were in grades 6-12 $(M=8.20, S D=1.88)$, with $405(72.5 \%)$ students in grades 6-8 and $187(27.5 \%)$ students in grades 9-12. The racial/ethnic distribution of the sample included Whites (77.9\%), Hispanics (4.6\%), Native Americans (3.5\%), African Americans (3.2\%), and others (10.3\%). 


\subsection{Instrument}

A test anxiety measure, the Test Anxiety Measure for Adolescents (TAMA; Lowe, 2014), was used in the present study. The TAMA consists of 44 items and includes five test anxiety scales. The Physiological Hyperarousal scale measures the physical symptoms associated with test anxiety and the Task Irrelevant Behaviors scale assesses the restless and avoidant behaviors exhibited in testing situations. The Social Concerns scale measures concerns that one will be ridiculed by others if poor test performance occurs and the Worry scale assesses concerns about failing a test and its repercussions. The Cognitive Interference scale measures the difficulty focusing on a test due to distracting thoughts dividing one's attention. A student uses a 4-point response scale to respond to the items. The response options range from $1=$ never to $4=$ almost always (Lowe, 2014). Coefficient alphas of .75 to .87 were reported for the TAMA scores in the current study. Validity evidence supporting the internal structure and external relations of the TAMA scores has been found (Lowe, 2014).

\subsection{Procedures}

This study was part of a larger study where the TAMA was completed along with other anxiety measures. The questionnaires were completed by the students in their schools. Parent consent and student assent was obtained as well as institutional review board approval before the study began. Students were instructed to read the directions on the measures and complete the questionnaires.

\subsection{Data Analysis}

LPAs were conducted using Mplus 7.11 software (L. K. Múthen \& B. O. Múthen, 1998-2013) with the robust maximum likelihood (MLR) estimator serving as the parameter estimator. TAMA scale scores were used as the indicators in the LPAs performed. A combination of fit indices, Akaike Information Criterion (AIC), Bayesian Information Criterion (BIC), Sample Adjusted BIC (SABIC), and Lo-Mendell-Rubin Likelihood Ratio Test (LMR LRT), along with entropy values as well as the interpretability of the LPA results were used to determine the optimal profile model, which consisted of a specific number of latent classes or subgroups (Muthén, 2003, Nylund, Asparouhov, \& Muthén, 2007) for the TAMA scales. Lower AIC, BIC, and SABIC values indicate a better model fit; however, the magnitude of the difference of the same criterion values across models should also be considered (Ferguson, Moore, \& Hull, 2020; Masyn, 2013). In addition, a nonsignificant LMR LRT when comparing two adjacent models indicates the parsimonious model has the better model fit (Ferguson, Moore, \& Hull, 2020). Finally, entropy was examined and a higher entropy value would suggest more certainty in one's classification (Wang, Bi, Ye, Deng, \& Yang, 2017).

After running the LPAs and identifying the best fitting model, gender (male, female) and grade-level (middle school, grades 6-8, high school, grades 9-12) were added to the model as covariates and logistic regression was performed to see if gender and/or grade-level could differentially predict membership in any of the latent classes or subgroups. Gender and grade-level were regressed on the latent classes. Any significant regression coefficient found would indicate a different likelihood for males and females or middle school students and high school students belonging to a specific latent test anxiety subgroup in comparison to another latent test anxiety subgroup.

\section{Results}

LPAs were performed on the TAMA and three models, two-, three-, and four-profile test anxiety models, were examined. The fit indices along with entropy values for the three different test anxiety profile models are presented in Table 1. The AIC, BIC, and SABIC were the lowest for the four-profile test anxiety model. In contrast, the highest entropy value was found for the two-profile test anxiety model. Furthermore, the LMR LRT was significant for the two-profile and three-profile test anxiety models. Based on these findings, the three-profile test anxiety model was selected as the best fitting model because this model had the second lowest AIC, BIC, and SABIC values, the second highest entropy value, and a significant LMR LRT value. According to the results, the addition of classes improved from two to three classes, but the four-class model did not fit better than the three-class model due to the nonsignificant LMR LRT value and parsimony. In addition, the selection of a three-class model is in agreement with von der Embse et al.'s (2014) LPA findings.

Average latent class probabilities for the three-profile test anxiety model are presented in Table 2. These probabilities indicate the greatest likelihood of a student belonging to its own class as well as each of the other classes. Examination of the probabilities in Table 2 indicated a student's greatest likelihood of belonging to its own class was quite high (greater than .92). These average posterior class probabilities support distinct classes. In contrast, the student's likelihood of belonging to any of the other classes was quite low (less than .06). These cross-probabilities indicate errors in classification are low. 
Table 1. Fit indices along with entropy values for the latent profile analysis for middle school and high school students on the test anxiety measure for adolescents

\begin{tabular}{cccccc}
\hline Model & AIC & BIC & SABIC & Entropy & LMR LRT \\
\hline 2-profile model & 17733.600 & 17803.736 & 17752.941 & .892 & $1304.514 * * * *$ \\
3-profile model & 17315.791 & 17412.228 & 17342.385 & .867 & $418.872 * * * *$ \\
4-profile model & 17228.109 & 17350.847 & 17261.956 & .866 & 97.146 \\
\hline
\end{tabular}

Note. SABIC $=$ Sample Adjusted Bayesian Information Criterion; AIC $=$ Akaike Information Criterion; BIC=Bayesian Information Criterion; LMR LRT $=$ Lo-Mendell-Rubin Likelihood Ratio Test; ${ }^{* * * *} p<.0001$.

Table 2. Average latent class probabilities on the test anxiety measure for adolescents

\begin{tabular}{cccc}
\hline Class & 1 & 2 & 3 \\
\hline 1 & .945 & .055 & .000 \\
2 & .055 & .921 & .024 \\
3 & .000 & .038 & .962 \\
\hline
\end{tabular}

Means were standardized with a variance of 1.00 for the different dimensions of test anxiety in the three-profile test anxiety model and are presented in Figure 1. In addition, independent $t$-tests were performed to compare the means of each dimension of test anxiety across subgroups. All $t$-tests were significant $\left(t_{s}>3.58, p<.001\right)$ indicating the class levels were different from each other. Subgroup 1 had the lowest test anxiety means and was named the Low Test Anxiety subgroup. The Low Test Anxiety subgroup included 272 (45.95\%) of the students. Within the Low Test Anxiety subgroup, Social Concerns had the lowest mean, followed by the Cognitive Interference mean, then the Worry mean, next the Physiological Hyperarousal mean, and finally the Task Irrelevant Behaviors mean. Subgroup 2 had test anxiety means in the middle range and was named the Medium Test Anxiety subgroup. The Medium Test Anxiety subgroup consisted of 210 (35.47\%) of the students. Within the Medium Test Anxiety subgroup, Social Concerns had the lowest mean, followed by the Task Irrelevant Behaviors mean, then the Physiological Hyperarousal mean, next the Cognitive Interference mean, and finally the Worry mean. Subgroup 3 had the highest test anxiety means and was named the High Test Anxiety subgroup. The High Test Anxiety subgroup included 110 (18.58\%) of the students. Within the High Test Anxiety subgroup, Social Concerns had the lowest mean, followed by the Task Irrelevant Behaviors mean, then the Cognitive Interference mean, next the Worry mean, and finally the Physiological Hyperarousal mean. 


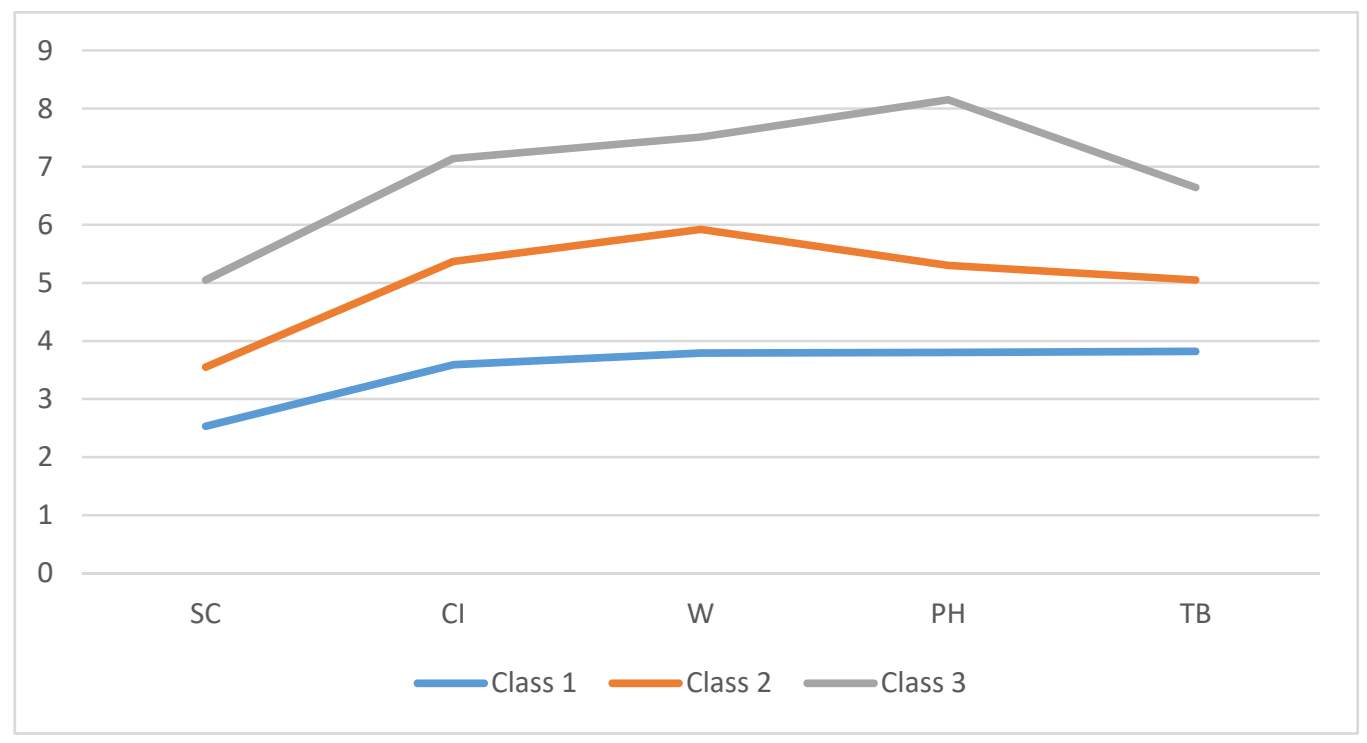

Figure 1. Three latent test anxiety subgroups

Note. Class 1=Low Test Anxiety Subgroup; Class 2=Medium Test Anxiety Subgroup; Class 3=High Test Anxiety Subgroup; $\mathrm{SC}=$ Social Concerns; $\mathrm{CI}=$ Cognitive Interference; $\mathrm{W}=$ Worry; $\mathrm{PH}=$ Physiological Hyperarousal; $\mathrm{TB}=$ Task Irrelevant Behaviors.

Gender and grade-level were entered as covariates in the three-profile test anxiety model and the model was run again to determine whether gender and grade-level could predict membership in the Low, Medium, and High Test Anxiety subgroups. Gender and grade-level differentially predicted class membership. Females were more likely to be in the High Test Anxiety subgroup (Odds Ratio $=1.60, S E=.32, p<.001$ ) than the Medium Test Anxiety subgroup and females were also more likely to be in the High Test Anxiety subgroup (Odds Ratio $=1.18, S E=.33$, $p<.001)$ than in the Low Test Anxiety subgroup. Middle school students were more likely to be in the High Test Anxiety subgroup (Odds Ratio $=.96, S E=.26, p<.001$ ) than the Low Test Anxiety subgroup and middle schools students were also more likely to be in the Medium Test Anxiety subgroup (Odds Ratio $=.60, S E=.23, p=.008$ ) than the Low Test Anxiety subgroup.

\section{Discussion and Conclusion}

Overall, the LPA findings in the present study indicated a three-profile test anxiety model was the best fitting model for U.S. secondary students. The three subgroups were named low, medium, and high test anxiety. These results are similar to von der Embse et al.'s (2014) findings with a group of only 11th grade U.S. students on a different test anxiety measure not developed in the United States. In von der Embse et al.'s pilot study, the authors found a three-profile test anxiety model as the best fitting model. Although limited research has been conducted using LPA in the area of test anxiety and additional research is needed, the findings from the current study and von der Embse et al.'s study suggest a three-profile model may be the best model for U. S. adolescent students when multidimensional measures of test anxiety are used.

Gender and grade-level differences were also examined to determine whether they differentially predicted membership in the three different latent test anxiety subgroups in the present study. More specifically, the author found females were more likely to be in the high latent test anxiety subgroup than in the medium and low latent test anxiety subgroups. These findings are in agreement with non-LPA studies of Brandmo, Bråten, and Schewe (2019) and von der Embse et al. (2018) where females were reported to have higher test anxiety levels than males. In von der Embse et al.'s (2018) study, the authors noted significant associations between gender and test anxiety in middle school students in grades 6-8 $(r=.16)$ and high school students in grades 9-12 $(r=.24$ to .25$)$, with females reporting higher levels of test anxiety than males. Moreover, in the current LPA study, middle school students were more likely to be found in the high and middle latent test anxiety subgroups than in the low latent test anxiety subgroup. These findings are in agreement with von der Embse et al.'s (2018) and Wigfield and Eccles's (1989) non-LPA results indicating that test anxiety tends to be at its highest level during the middle school/junior high school years. 
There are theoretical and practical implications associated with the results of the present study. The findings of the current study clearly identified three test anxiety profiles, supporting test anxiety as a multidimensional construct. The multidimensionality of the test anxiety construct is in agreement with Lowe et al.'s (2008) biopsychosocial model of test anxiety where a student's test anxiety is expressed in the form of cognitive, behavioral, and physical symptoms. The TAMA was developed based on the biopsychosocial model of test anxiety. Second, the present study identified low, medium, and high subgroups of test-anxious adolescents on a test anxiety measure. In the high latent test anxiety subgroup, physiological hyperarousal symptoms were the highest, followed by worry symptoms, and then cognitive interference symptoms, with task irrelevant behaviors and social concerns symptoms being lower. Although additional research is needed, elevated symptoms of test anxiety exhibiting a similar pattern in adolescents may suggest that these individual adolescents may be experiencing clinical levels of test anxiety and professionals may be able to assist and implement interventions to reduce their test anxiety. Cognitive symptoms of test anxiety have been found to be more associated with poor test performance than physiological hyperarousal symptoms (Cassady, 2004), and cognitive-behavioral strategies have been reported to reduce test anxiety in the adolescent population (de Hullu, Sportel, Esther, Maaike, \& de Jong, 2017; Putwain \& Pescod, 2018; von der Embse, Barterian, \& Segool, 2013). However, addressing physiological hyperarousal symptoms, when cognitive symptoms are also experienced have been found to be beneficial for those individuals who do experience test anxiety (Schwarzer, 1984; Schwarzer \& Jerusalem, 1992). Relaxation strategies can address and have been reported to reduce the physiological hyperarousal symptoms of test anxiety in adolescent students (Tseng \& Wang, 2011; von der Embse et al., 2013). As for the behavioral symptoms, these symptoms could potentially be addressed through problem-focused coping strategies and relaxation strategies (Zeidner, 1998). Furthermore, although test anxiety can affect male and female adolescents and middle school and high school students, the findings from the present study suggest that female adolescents and middle school students are possibly at a greater risk of developing test anxiety. Therefore, schools should keep this in mind in planning and implementing prevention strategies to prevent test anxiety symptoms in the adolescent population.

There are several limitations associated with the present study. The sample used in the current study was a sample of convenience. It would be important in future studies to replicate the present study with an ethnically and regionally diverse sample of adolescents from the United States and determine whether similar results would be found. Another limitation was only a self-report measure of test anxiety was used in the present study. In future studies, behavioral rating scales assessing test anxiety completed by other informants who are knowledgeable about the participants would be helpful to include. The inclusion of behavioral rating scales completed by informants would provide additional information on each participant's test anxiety and would also reduce the potential for possible method bias.

There are other areas for future research with the TAMA and the adolescent population. Additional LPA studies should be conducted with the TAMA to examine the relation between different latent test anxiety subgroups and academic achievement measures in adolescent students to determine whether students in the high latent test anxiety subgroup perform lower on measures of academic performance. In addition, the relationship between test anxiety and other types of anxiety, such as social anxiety, performance anxiety, general anxiety, and math anxiety using LPA procedures would be helpful in understanding whether test anxiety is more closely related to social anxiety, general anxiety, math anxiety, or performance anxiety. Finally, the relationship between different latent test anxiety subgroups and different dimensions of perfectionism could be investigated using the TAMA and LPA procedures. The findings from these latter LPA studies may add clarity to the complex relationship between test anxiety and perfectionism.

In sum, the LPA results identified three anxiety profiles on a test anxiety measure designed for use with adolescent students in the United States. This finding supports the multidimensionality of the test anxiety construct. The three anxiety profiles identified in the current study were named high, medium, and low test anxiety. The high latent test anxiety subgroup had the highest physiological hyperarousal symptoms, followed by worry symptoms, and then cognitive interference symptoms, with lower task irrelevant behaviors and social concern symptoms. Furthermore, females and middle school students were more likely to be found in the high latent test anxiety subgroup in comparison to males and high school students, respectively. These findings have potential implications for the development of test anxiety measures, and the treatment and prevention of test anxiety in the U.S. adolescent population.

\section{References}

Bodas, J., \& Ollendick, T. H. (2005). Test anxiety: A cross-cultural perspective. Clinical Child and Family Psychology Review, 8, 65-88. https://doi.org/10.1007/s10567-005-2342-x 
Bodas, J., Ollendick, T. H., \& Sovani, A. V. (2008). Test anxiety in Indian children: A cross-cultural perspective. Anxiety, Stress, \& Coping, 21, 387-404. https://doi.org/10.1080/10615800701849902

Brandmo, C., Bråten, I., \& Schewe, O. (2019). Social and personal predictors of test anxiety among Norwegian secondary and postsecondary students. Social Psychology of Education: An International Journal, 22, 43-61. https://doi.org/10.1007/s11218-018-9461-y

Carey, E., Devine, A., Hill, F., \& Szücs, D. (2017). Differentiating anxiety forms and their role in academic performance from primary to secondary school. PLOS ONE, 12(3), e0174418. https://doi.org/10.1371/journal.pone.0174418

Cassady, J. C. (2004). The influence of cognitive test anxiety across the learning-testing cycle. Learning and Instruction, 14, 569-592. https://doi.org/10.1016/j.learninstruc.2004.09.002

Chapell, M. S., Blanding, Z. B., Silverstein, M. E., Takahashi, M. N. B., Newman, B., Gubi, A., \& McCain, N. (2005). Test anxiety and academic performance in undergraduate and graduate students. Journal of Educational Psychology, 97, 268-274. https://doi.org/10.1037/0022-0663.97.2.268

Cizek, G. J., \& Burg, S. S. (2006). Addressing test anxiety in a high-stakes environment. Thousand Oaks, CA: Corwin Press.

Collins, I. M., \& Lanza, S. T. (2013). Latent class and latent transition analysis: With applications in the social, behavioral, and health sciences. Hoboken, NJ: John Wiley \& Sons.

Costa, P. T., Herbst, J. H., McCrae, R. R., Samuels, J., \& Ozer, D. J. (2002). The replicability and utility of three personality types. European Journal of Personality, 16, 73-87. https://doi.org/10.1002/per.448

De Hullu, E., Sportel, B., Esther, N., Maaike, H., \& de Jong, P. J. (2017). Cognitive bias modification and CBT as early interventions for adolescents social and test anxiety: Two-year follow-up of a randomized controlled trial. Journal of Behavior Therapy and Experimental Psychiatry, 55, 81-89. https://doi.org/j.jbtep.2016.11.0111

Every Student Succeeds Act. (2015). Public Law No. 114-95, 129 Stat. 1802.

Ferguson, S. L., Moore, E. W., \& Hull, D. M. (2020). Finding latent groups in observed data: A primer on latent profile analysis in Mplus for applied researchers. International Journal of Behavioral Development, 44, 458-468. https://doi.org/10.1177/0165025419881721

Jacob, S., Decker, D. M., \& Timmerman Lugg, E. (2016). Ethics and law for school psychologists (7th ed.). Hoboken, NJ: Wiley.

Knappe, S., Beesdo-Baum, K., Fehm, L., Stein, M. B., Lieb, R., \& Wittchen, H. U. (2011). Social fear and social phobia types among community youth: Differential clinical features and vulnerability factor. Journal of Psychiatric Research, 45, 111-120. https://doi.org/10.1016/j.jpsychires.2010.05.002

Lowe, P. A. (2014). The Test Anxiety Measure for Adolescents (TAMA): Examination of the reliability and validity of the scores of a new multidimensional measure of test anxiety for middle and high school students. Journal of Psychoeducational Assessment, 32, 404-416. https://doi.org/10.1177/0734282913520595

Lowe, P. A., Lee, S. W., Witteborg, K. M., Prichard, K. W., Luhr, M. E., Cullinan, C. M., ... Janik, M. (2008). The Test Anxiety Inventory for Children and Adolescents (TAICA): Examination of the psychometric properties of a new multidimensional measure of test anxiety among elementary and secondary school students. Journal of Psychoeducational Assessment, 26, 230. https://doi.org/10.1177/0734282907303760

Mammarella, I. C., Donolato, E., Caviola, S., \& Giofré, D. (2018). Anxiety profiles and protective factors: A latent profile analysis in children. Personality and Individual Differences, 124, 201-208. https://doi.org/10.1016/j.paid.2017.12.017

Masyn, K. E. (2013). Latent class analysis and finite mixture modeling. In T. Little (Eds.), The Oxford handbook of quantitative methods (pp. 551-611). New York: Oxford University Press. https://doi.org/10.1093/oxfordhb/9780199934898.013.0025

Mowbray, T. (2012). Working memory, test anxiety and effective interventions: A review. The Australian Educational and Developmental Psychologist, 29, 141-156. https://doi.org/10.1017/edp.2012.16

Muthén, B. O. (2003). Statistical and substantive checking in growth mixture modeling: Comment on Bauer and Curran (2003). Psychological Methods, 8, 369-377. https://doi.org/10.1037/1082-989X.8.3.369 
Muthén, L. K., \& Muthén, B. O. (1998-2013). Mplus version 7.11. Los Angeles, CA: Author.

Nylund, K. L., Asparouhov, T., \& Muthén, B. O. (2007). Deciding on the number of classes in latent class analysis and growth mixture modeling: A Monte Carlo simulation study. Structural Equation Modeling: A Multidisciplinary Journal, 14, 535-569. https://doi.org/10.1080/10705510701575396

Putwain, D. W., \& Daly, A. L. (2014). Test anxiety prevalence and gender differences in a sample of English $\begin{array}{lllll}\text { secondary school students. } & \text { Educational }\end{array}$ https://doi.org/10.1080/03055698.2014.953914

Putwain, D. W., \& Pescod, M. (2018). Is reducing uncertain control the key to successful test anxiety intervention for secondary school students? Findings from a randomized control trail. School Psychology Quarterly, 33, 283-292. https://doi.org/10.1037/spq0000228

Ringeisen, T., \& Raufelder, D. (2015). The interplay of parental support, parental pressure and test anxiety: Gender differences in adolescents. Journal of Adolescence, 45, 67-79. https://doi.org/10.1016/j.adolescence.2015.08.018

Schwarzer, R. (1984). Worry and emotionality as separate components in test anxiety. International Review of Applied Psychology, 33, 205-220. https://doi.org/10.1111/j.1464-0597.1984.tb01429.x

Schwarzer, R., \& Jerusalem, M. (1992). Advances in anxiety theory: A cognitive process approach. In K. A. Hagtvet \& T. B. Johnsen (Eds.), Advances in test anxiety research (Vol. 7, pp. 2-31). Lisse, Netherlands: Swets \& Zeitlnger.

Spurk, D., Hirschi, A., Wang, M., Valero, D., \& Kauffeld, S. (2020). Latent profile analysis: A review and "how to" guide of its application within vocational behavior research. Journal of Vocational Behavior, 120. https://doi.org/10.1016/j.jvb.2020.103445

Tseng, Y., \& Wang, M. L. (2011). The application of guided imagery to reduce high school students' test anxiety. Journal of Youth Studies, 14, 98-110.

Vermunt, J. K., \& Magidson, J. (2002). Latent class cluster analysis. In J. Hagenaars, \& A. McCutcheon (Eds.), Applied latent class analysis (Vol. 11, pp. 89-106). Cambridge: Cambridge University Press. https://doi.org/10.1017/CBO9780511499531.004

Von der Embse, N. P., Mata, A. D., Segool, N., \& Scott, E.C. (2014). Latent profile analysis of test anxiety: A

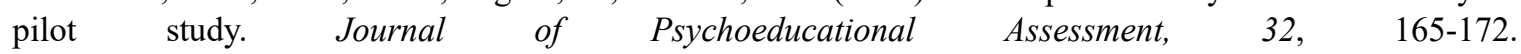
https://doi.org/10.1177/0734282913504541

Von der Embse, N., Barterian, J., \& Segool, N. (2013). Test anxiety interventions for children and adolescents: A systematic review of treatment studies from 2000-2010. Psychology in the Schools, 50, 57-71. https://doi.org/10.1002/pits.21660

Von der Embse, N., Jester, D., Roy, D., \& Post, J. (2018). Test anxiety effects, predictions, and correlations: A 30 year meta-analysis review. Journal of Affective Disorders, 227, 483-493. https://doi.org/10.1016/j.jad.2017.11.048

Wang, M., \& Hanges, P. J. (2011). Latent class procedures: Applications to organizational research. Organizational Research Methods, 14, 24-31. https://doi.org/10.1177/1094428110383988

Wang, M., Deng, Q., Bi, X., Ye, H., \& Yang, W. (2017). Performance of the entropy index of classification accuracy in latent profile analysis: A Monte Carlo simulation study. Acta Psychlogica Sinica, 49, 1473-1482. https://doi.org/10.3724/SP.J.1041.2017.01473

Wigfield, A., \& Eccles, J. S. (1989). Test anxiety in elementary and secondary school students. Educational Psychologist, 24, 159-183. https://doi.org/10.1207/s15326985ep2402_3

Wild, E., Hofer, M., \& Pekrun, R. (2006). Psychologie des lerners [The psychology of the learners]. In A. Krapp \& B. Weidenmann (Eds.), Padagogische psychologie. Ein lehrbuch (Vol. 5, pp. 203-265). Weinheim, Germany: Beltz.

Woo, S. E., Jebb, A. T., Tay, L., \& Parrigon, S. (2018). Putting the "person" in the center: Review and synthesis of person-centered approaches and methods in organizational science. Organizational Research Methods, 4, 814-845. https://doi.org/10.1177/1094428117752467

Yousefi, F. (2012). Depression, test anxiety, memory and academic achievement. Saarbrucken, Germany: LAP Lambert. 
Zeidner, M. (1998). Test anxiety: The state of the art. New York: Plenum.

Zeidner, M. (2014). Anxiety in education. In R. Pekrun, \& L. Linnenbrink-Garcia (Eds.), International handbook of emotions in education (pp. 265-288). New York: Routledge.

\section{Copyrights}

Copyright for this article is retained by the author(s), with first publication rights granted to the journal.

This is an open-access article distributed under the terms and conditions of the Creative Commons Attribution license (http://creativecommons.org/licenses/by/4.0/). 